\title{
Sequences of Tridovan and their identities
}

\author{
Renata Passos Machado Vieira ${ }^{1}$ \\ and Francisco Regis Vieira Alves ${ }^{2}$
}

\author{
${ }^{1}$ Department of Mathematics \\ Institute Federal of Technology of the State of Ceará (IFCE) \\ Fortaleza-CE, Brazil \\ e-mail: re.passosm@gmail.com \\ ${ }^{2}$ Department of Mathematics \\ Institute Federal of Technology of the State of Ceará (IFCE) \\ Fortaleza-CE, Brazil \\ e-mail: fregis@gmx.fr
}

Received: 19 December $2018 \quad$ Revised: 20 June $2019 \quad$ Accepted: 29 July 2019

\begin{abstract}
This work introduces the so-called Tridovan sequence which is an extended form of the Padovan sequence. In a general definition, this extension adds one more term to the Padovan recurrence relation, considering now the three terms preceding the penultimate one. Studies carried out on the proposed extension reveal properties of the positive and negative integer index, the sum of all, even and odd terms, the obtaining Tridovan Q-matrix and finally the Tridovan initial terms generalization.
\end{abstract}

Keywords: Sequence of Tridovan, Positive indices, Negative indices, Generating matrix.

2010 Mathematics Subject Classification: 11B37, 11 B39.

\section{Introduction}

The Italian architect Richard Padovan (born 1935) discovered a kind of "cousin" of the well-known Fibonacci sequence, the Padovan numbers, which is also recursive, arithmetic and linear. Padovan was born in the city of Padua [12], a village a little far from the same birthplace of Fibonacci [1].

The Padovan sequence is defined by the recursive relation $P_{n}=P_{n-2}+P_{n-3}, n \geq 3$, where $P_{n}$ is the $n$-th term of the sequence. The first sequence terms are defined by $P_{0}=P_{1}=P_{2}=1$, which may generate the following initial elements: $1,1,1,2,2,3,4,5,7, \ldots$. 
An important feature of this sequence is the neighboring terms relationship $P_{n+1} / P_{n}$, which converges to a value known as plastic number or plastic constant $[8,10]$. The approximate value of the plastic number is $\psi=1.324718 \ldots$ and the ways of calculating this value are presented in the $[6,8]$.

Based on the Fibonacci sequence, [4, 9] proposed a sequence extension considering not only the sum of two previous elements but three or four previous elements. Following the same path it is possible to extend the Padovan numbers considering a greater number of terms to estimate the next one.

The geometric representation of the Padovan sequence occurs through the Padovan spiral, as shown in Figure 1. This is composed by the juxtaposition of equilateral triangles respecting a characteristic construction rule. Consider the side 1 highlighted triangle in blue as the initial triangle. The spiral formation of the spiral is given by the addition of a new equilateral triangle to the largest side of the formed polygon, initially the blue triangle. After the addition of the other triangles, the new polygon is formed, known as plastic pentagon. The spiral presents itself by connecting with an arc the two corners of the newly added triangle.

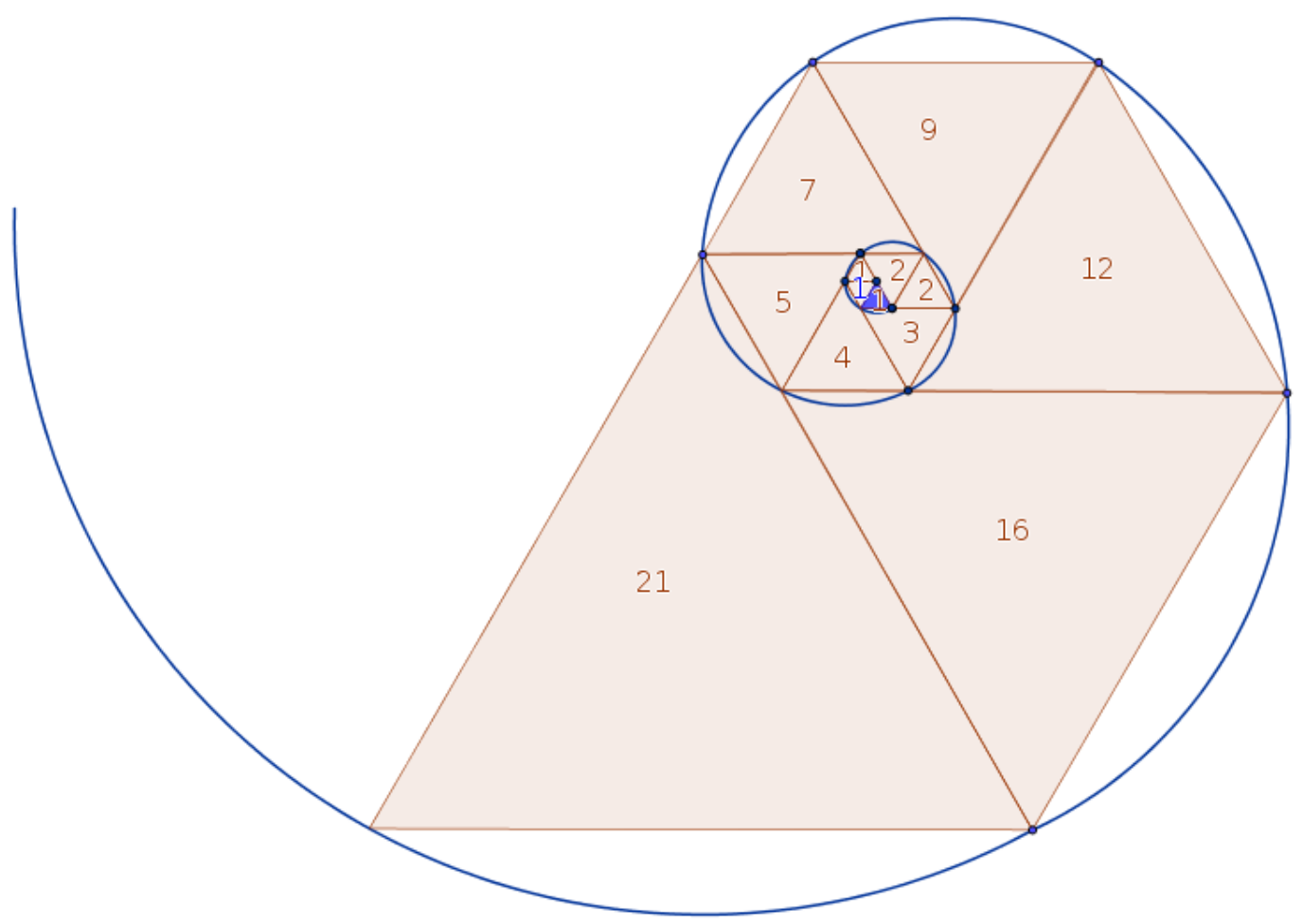

Figure 1. Padovan spiral. (Source: Prepared by the authors.)

Although this sequence is recursive, its terms can be found through the Q-matrix. According to studies carried out by Alves [2] for the Fibonacci sequence, the same idea will be followed so that an application is made for the Tridovan numbers. Thus, it is possible to study these new identities in addition to providing the perception of a constantly evolving notion of this sequence. 


\section{Preliminary}

As defined in the previous section, any element of the Padovan sequence is calculated based on the sum of the last two terms ignoring the one immediately before. Following the same idea, one can define the Tridovan sequence as the sum of the tree last terms ignoring the one immediately before. The addition of this new element makes the Tridovan Sequence a $4^{\text {th }}$ degree recurrent linear sequence, and its properties and definitions will be presented in the following sessions.

Definition 1. The Tridovan sequence numbers recursive relation for positive indices is given by:

$$
T_{n}=T_{n-2}+T_{n-3}+T_{n-4}, n \geq 4,
$$

where $T_{n}$ is the $n$-th term of the sequence. The initial values of these numbers are given by: $T_{0}=0, T_{1}=1, T_{2}=0, T_{3}=1$. Thus, the list of terms in this sequence are $(0,1,0,1,1,2,2,4,5$, $8,11,17, \ldots)$.

Definition 2. The Tridovan sequence recurrence relation for negative indices is given by: $T_{-n}=T_{-n+4}-T_{-n+2}-T_{-n+1}$ or $T_{-n}=T_{-n+4}-T_{-n+2}+T_{-n+1}$, with $T_{0}=0, T_{1}=1, T_{2}=0, T_{3}=1$.

From Definition 1, we can naturally describe the Tridovan sequence. We can also observe a process of extension of integer indices, since, in the previous definition, its values are indicated only for $n \geq 4$. So let us see that for $n=3 \therefore T_{3}=T_{1}+T_{0}+T_{-1}$ and, thus, we will have the $T_{-1}=0$. Similarly, we see that for $n=2 \therefore T_{2}=T_{0}+T_{-1}+T_{-2}$ and, we will have the value $T_{-2}=0$. For a smaller index, we have $n=1 \therefore T_{1}=T_{-1}+T_{-2}+T_{-3}$, getting $T_{-3}=1$. On a recurrent basis, we can further determine that $T_{-4}=-1, T_{-5}=0, T_{-6}=1, T_{-7}=0, T_{-8}=-2, T_{-9}=2, T_{-10}=1, T_{-11}=-3$, etc. With this, from the previous values, we can systematize the description of the following sets with integers, as follows:

$$
\left(\ldots, T_{-n-2}, T_{-n-1}, T_{-n}, T_{-n+1}, T_{-n+2}, \ldots T_{-4}, T_{-3}, T_{-2}, T_{-1}, T_{0}, T_{1}, T_{2}, T_{3}, T_{4}, \ldots, T_{n-3}, T_{n-2}, T_{n-1}, T_{n}, \ldots\right) .
$$

When we want to determine any element for $n \geq 4$, we use the relationship $T_{n}=T_{n-2}+T_{n-3}+T_{n-4}$, however, the elements $T_{0}=0, T_{1}=1, T_{2}=0, T_{3}=1$ are fixed preliminarily.

Now, noticing that $T_{-1}=T_{3}-T_{1}-T_{0}$ or yet $T_{-2}=T_{2}-T_{0}-T_{-1}$ and also $T_{-3}=T_{1}-T_{-1}-T_{-2}$, constructing: $(\ldots,-3,1,2,-2,0,1,0,-1,1,0,0,0,1,0,1,1,2,2,4, \ldots)$ we can write the relation of recurrence to negative indexes as: $T_{-n}=T_{-n+4}-T_{-n+2}-T_{-n+1}$ or $T_{-n}=T_{-n+4}-T_{-n+2}+T_{-n+1}$.

This arrangement shows a different understanding of the one shown at the beginning, since before the development of the terms were found by performing a "movement" from left to right, these terms can now be found with right-to-left "movement") of the sequence.

Definition 3. From the Tridovan sequence, we use the following notations with initial values $T_{0}=0, T_{1}=1, T_{2}=0, T_{3}=1$, and $S_{0}=0, S_{1}=1, S_{2}=1, S_{3}=2$, and:

(a) Sum of positive terms: $S_{n}=T_{0}+T_{1}+T_{2}+\ldots+T_{n-1}+T_{n}=\sum_{i=0}^{n} T_{i}$,

(b) Sum of positive even-numbered terms: $S_{2 n}=T_{0}+T_{2}+T_{4}+\ldots+T_{2 n-2}+T_{2 n}=\sum_{i=0}^{n} T_{2 i}$, 
(c) Sum of positive odd-numbered terms: $S_{2 n+1}=T_{1}+T_{3}+T_{5}+\ldots+T_{2 n-1}+T_{2 n+1}=\sum_{i=0}^{n} T_{2 i+1}$,

(d) Sum of negative terms: $S_{-n}=T_{0}+T_{-1}+T_{-2}+\ldots+T_{-n-1}+T_{-n}=\sum_{i=0}^{n} T_{-i}$,

(e) Sum of negative even-numbered terms: $S_{-2 n}=T_{0}+T_{-2}+T_{-4}+\ldots+T_{-2 n-2}+T_{-2 n}=\sum_{i=0}^{n} T_{-2 i}$,

(f) Sum of negative odd-numbered terms: $S_{-2 n-1}=T_{-1}+T_{-3}+T_{-5}+\ldots+T_{-2 n-1}=\sum_{i=0}^{n} T_{-2 i-1}$.

Theorem 1. For any integer $n \geq 2$ the following identities are verified:

(i) $S_{n}+S_{n-1}=T_{n+3}-1$;

(ii) $S_{2 n}+S_{2 n-1}=T_{2 n+3}-1$,

(iii) $S_{2 n-1}+S_{2 n-2}=T_{2 n+2}$.

Proof. Consider the following set of expressions:

$$
\left\{\begin{array} { l } 
{ T _ { 4 } = T _ { 2 } + T _ { 1 } + T _ { 0 } } \\
{ T _ { 5 } = T _ { 3 } + T _ { 2 } + T _ { 1 } } \\
{ T _ { 6 } = T _ { 4 } + T _ { 3 } + T _ { 2 } } \\
{ \vdots } \\
{ T _ { n + 1 } = T _ { n - 1 } + T _ { n - 2 } + T _ { n - 3 } } \\
{ T _ { n + 2 } = T _ { n } + T _ { n - 1 } + T _ { n - 2 } } \\
{ T _ { n + 3 } = T _ { n + 1 } + T _ { n } + T _ { n - 1 } }
\end{array} \quad \therefore \left\{\begin{array}{l}
T_{4}-T_{2}=T_{1}+T_{0} \\
T_{5}-T_{3}=T_{2}+T_{1} \\
T_{6}-T_{4}=T_{3}+T_{2} \\
\vdots \\
T_{n+1}-T_{n-1}=T_{n-2}+T_{n-3} \\
T_{n+2}-T_{n}=T_{n-1}+T_{n-2} \\
T_{n+3}-T_{n+1}=T_{n}+T_{n-1}
\end{array}\right.\right.
$$

We can effectively cancel out the similar terms in the above equations to determine the following sum

$$
T_{n+3}-T_{2}-T_{3}=\sum_{i=0}^{n} T_{i}+\sum_{i=0}^{n-1} T_{i},
$$

remembering that $T_{2}=0, T_{3}=1$ and, therefore, we see that

$$
S_{n}+S_{n-1}=\sum_{i=0}^{n} T_{i}+\sum_{i=0}^{n-1} T_{i}=T_{n+3}-1,
$$

or even more simplified as $S_{n}+S_{n-1}=T_{n+3}-1$, hence we prove (i).

In a similar way, this time for the even indices, we have the following set of equations:

$$
\left\{\begin{array} { l } 
{ T _ { 5 } = T _ { 3 } + T _ { 2 } + T _ { 1 } } \\
{ T _ { 7 } = T _ { 5 } + T _ { 4 } + T _ { 3 } } \\
{ T _ { 9 } = T _ { 7 } + T _ { 6 } + T _ { 5 } } \\
{ \vdots } \\
{ T _ { 2 n - 1 } = T _ { 2 n - 3 } + T _ { 2 n - 4 } + T _ { 2 n - 5 } } \\
{ T _ { 2 n + 1 } = T _ { 2 n - 1 } + T _ { 2 n - 2 } + T _ { 2 n - 3 } } \\
{ T _ { 2 n + 3 } = T _ { 2 n + 1 } + T _ { 2 n } + T _ { 2 n - 1 } }
\end{array} \quad \therefore \left\{\begin{array}{l}
T_{5}-T_{3}=T_{2}+T_{1} \\
T_{7}-T_{5}=T_{4}+T_{3} \\
T_{9}-T_{7}=T_{6}+T_{5} \\
\vdots \\
T_{2 n-1}-T_{2 n-3}=T_{2 n-4}+T_{2 n-5} \\
T_{2 n+1}-T_{2 n-1}=T_{2 n-2}+T_{2 n-3} \\
T_{2 n+3}-T_{2 n+1}=T_{2 n}+T_{2 n-1}
\end{array}\right.\right.
$$

By means of cancelling out, we can obtain: 


$$
T_{2 n+3}-T_{3}=T_{2 n+3}-1=\sum_{i=1}^{n} T_{2 i}+\sum_{i=0}^{n-1} T_{2 i+1}
$$

assuming $T_{3}=1$, we find that $T_{2 n+3}-1=S_{2 n}+S_{2 n-1}$, hence we prove (ii).

We can also consider the following arrangement of the elements of the first list, eliminating the odd indexes as follows:

$$
\left\{\begin{array} { l } 
{ T _ { 4 } = T _ { 2 } + T _ { 1 } + T _ { 0 } } \\
{ T _ { 6 } = T _ { 4 } + T _ { 3 } + T _ { 2 } } \\
{ T _ { 8 } = T _ { 6 } + T _ { 5 } + T _ { 4 } } \\
{ T _ { 1 0 } = T _ { 8 } + T _ { 7 } + T _ { 6 } } \\
{ \vdots } \\
{ T _ { 2 n } = T _ { 2 n - 2 } + T _ { 2 n - 3 } + T _ { 2 n - 4 } } \\
{ T _ { 2 n + 2 } = T _ { 2 n } + T _ { 2 n - 1 } + T _ { 2 n - 2 } }
\end{array} \quad \therefore \left\{\begin{array}{l}
T_{4}-T_{2}=T_{1}+T_{0} \\
T_{6}-T_{4}=T_{3}+T_{2} \\
T_{8}-T_{6}=T_{5}+T_{4} \\
T_{10}-T_{8}=T_{7}+T_{6} \\
\vdots \\
T_{2 n}-T_{2 n-2}=T_{2 n-3}+T_{2 n-4} \\
T_{2 n+2}-T_{2 n}=T_{2 n-1}+T_{2 n-2}
\end{array}\right.\right.
$$

Thus, considering the corresponding eliminations, we must find that

$$
T_{2 n+2}-T_{2}=\sum_{i=0}^{n-1} T_{2 n+1}+\sum_{i=0}^{n-1} T_{2 n}
$$

therefore, we can write

$$
T_{2 n+2}=\sum_{i=0}^{n-1} T_{2 n+1}+\sum_{i=0}^{n-1} T_{2 n}=S_{2 n-1}+S_{2 n-2}
$$

which proves item (iii).

Finally, let us note that $n=4 \therefore 1+S_{2}+S_{1}+S_{0}=1+1+1+0=3=S_{4}$. Using another value for $n$, one can get $n=5 \therefore 1+S_{3}+S_{2}+S_{1}=1+2+1+1=5=S_{5}$. Thus, through the inductive method, we can see that:

$$
S_{n}=1+S_{n-2}+S_{n-3}+S_{n-4}
$$

For $n=n+1$, we have

$$
\begin{gathered}
S_{n+1}=S_{n}+T_{n+1}=\left(1+S_{n-2}+S_{n-3}+S_{n-4}\right)+T_{n+1}=\left(1+S_{n-2}+S_{n-3}+S_{n-4}\right)+\left(T_{n-1}+T_{n-2}+T_{n-3}\right) \\
S_{n+1}=1+\left(S_{n-2}+T_{n-1}\right)+\left(S_{n-3}+T_{n-2}\right)+\left(S_{n-4}+T_{n-3}\right)=1+S_{n-1}+S_{n-2}+S_{n-3}
\end{gathered}
$$

Theorem 2. For any integer $n \geq 2$ the following identities are verified:

(i) $-T_{-2 n-2}=S_{-2 n-3}+S_{-2 n-4}$;

(ii) $-\left(T_{-n-2}+T_{-n-3}\right)=S_{-n-4}+S_{-n-5}$.

Proof. Let us consider the equations:

$$
\left\{\begin{array}{l}
T_{0}=T_{-2}+T_{-3}+T_{-4} \\
T_{-2}=T_{-4}+T_{-5}+T_{-6} \\
T_{-4}=T_{-6}+T_{-7}+T_{-8} \\
T_{-6}=T_{-8}+T_{-9}+T_{-10} \\
\vdots \\
T_{-2 n}=T_{-2 n-2}+T_{-2 n-3}+T_{-2 n-4}
\end{array}\right.
$$


Performing some algebraic manipulations, we have:

$$
\left\{\begin{array}{l}
T_{0}-T_{-2}=T_{-3}+T_{-4} \\
T_{-2}-T_{-4}=T_{-5}+T_{-6} \\
T_{-4}-T_{-6}=T_{-7}+T_{-8} \\
T_{-6}-T_{-8}=T_{-9}+T_{-10} \\
\vdots \\
T_{-2 n}-T_{-2 n-2}=T_{-2 n-3}+T_{-2 n-4}
\end{array} .\right.
$$

Adding and eliminating the corresponding terms, we find

$$
T_{0}-T_{-2 n-2}=\sum_{i=1}^{n+1} T_{-2 i-1}+\sum_{i=1}^{n+1} T_{-2 i-2}=-T_{-2 n-2}=\sum_{i=1}^{n+1} T_{-2 i-1}+\sum_{i=1}^{n+1} T_{-2 i-2}-T_{-2 n-2}=S_{-2 n-3}+S_{-2 n-4},
$$

which proves item (i).

Then, we will use the following set of relations

$$
\left\{\begin{array}{l}
T_{0}=T_{-2}+T_{-3}+T_{-4} \\
T_{-1}=T_{-3}+T_{-4}+T_{-5} \\
T_{-2}=T_{-4}+T_{-5}+T_{-6} \\
T_{-3}=T_{-5}+T_{-6}+T_{-7} \\
\vdots \\
T_{-n}=T_{-n-2}+T_{-n-3}+T_{-n-4} \\
T_{-n-1}=T_{-n-3}+T_{-n-4}+T_{-n-5}
\end{array}\right.
$$

Through simple algebraic manipulations, we obtain:

$$
\left\{\begin{array}{l}
T_{0}-T_{-2}=T_{-3}+T_{-4} \\
T_{-1}-T_{-3}=T_{-4}+T_{-5} \\
T_{-2}-T_{-4}=T_{-5}+T_{-6} \\
T_{-3}-T_{-5}=T_{-6}+T_{-7} \\
\vdots \\
T_{-n}-T_{-n-2}=T_{-n-3}+T_{-n-4} \\
T_{-n-1}-T_{-n-3}=T_{-n-4}+T_{-n-5}
\end{array}\right.
$$

With this, after cancelling out, we can see that:

$$
T_{0}+T_{1}-T_{-n-2}-T_{-n-3}=\sum_{i=3}^{n+4} T_{-i}+\sum_{i=4}^{n+5} T_{-i}
$$

Knowing that the values of $T_{0}=T_{-1}=0$, we have: $-\left(T_{-n-2}+T_{-n-3}\right)=S_{-n-4}+S_{-n-5}$, which proves item (ii).

Definition 4. Given the arbitrary numbers $G_{1}, G_{2}, G_{3}$, the Generalized Tridovan Sequence's recurrence relation is defined as following:

$$
G_{n}=G_{n-1}+G_{n-2}+G_{n-3} .
$$


Theorem 3. For any integer $n \geq 3$ will have the following general term

$$
G_{n}=\left(T_{n-3}+T_{n-4}\right) \cdot G_{1}+T_{n-1} \cdot G_{2}+T_{n-2} \cdot G_{3} .
$$

It is worth noting that $T_{n}$ represents the $n$-th term of the Tridovan Sequence (see Definition 1) and $G_{n}$ represents the $n$-th term of the Generalized Tridovan Sequence (see Definition 4).

Proof. Consider

$$
G_{4}=G_{2}+G_{1}+G_{0}=G_{1}+G_{2}=1 \cdot G_{2}+1 \cdot G_{2}=T_{3} \cdot G_{2}+T_{2} \cdot G_{3} .
$$

For

$$
G_{5}=G_{1}+G_{2}+G_{3}=1 \cdot G_{1}+1 \cdot G_{2}+1 \cdot G_{3}=\left(T_{1}+T_{2}\right) \cdot G_{1}+T_{4} \cdot G_{2}+T_{3} \cdot G_{3}
$$

Admitting the inductive step $G_{n}=\left(T_{n-4}+T_{n-3}\right) \cdot G_{1}+T_{n-1} \cdot G_{2}+T_{n-2} \cdot G_{3}$, we will add the following terms $G_{n+2}=G_{n}+\left(G_{n-1}+G_{n-2}\right)=\left(T_{n-3}+T_{n-4}\right) \cdot G_{1}+T_{n-1} \cdot G_{2}+T_{n-2} \cdot G_{3}+\left(G_{n-1}+G_{n-2}\right)$. In the next step, we have:

$$
\begin{aligned}
& G_{n-1}=\left(T_{n-4}+T_{n-5}\right) \cdot G_{1}+T_{n-2} \cdot G_{2}+T_{n-3} \cdot G_{3}, \\
& G_{n-2}=\left(T_{n-5}+T_{n-6}\right) \cdot G_{1}+T_{n-3} \cdot G_{2}+T_{n-4} \cdot G_{3} .
\end{aligned}
$$

And then replacing the terms, using the hypothesis:

$$
\begin{aligned}
G_{n+2}= & G_{n}+\left(G_{n-1}+G_{n-2}\right) \\
= & \left(T_{n-3}+T_{n-4}\right) \cdot G_{1}+T_{n-1} \cdot G_{2}+T_{n-2} \cdot G_{3}+\left(T_{n-4}+P_{n-5}\right) \cdot G_{1} \\
& +T_{n-2} \cdot S_{2}+T_{n-3} \cdot G_{3}+\left(T_{n-5}+T_{n-6}\right) \cdot G_{1}+T_{n-3} \cdot G_{2}+T_{n-4} \cdot G_{3} \\
= & \left(T_{n-3}+T_{n-4}+T_{n-4}+T_{n-5}+T_{n-5}+T_{n-6}\right) \cdot G_{1}+\left(T_{n-1}+T_{n-2}+T_{n-3}\right) \cdot G_{2}+\left(T_{n-2}+T_{n-3}+T_{n-4}\right) \cdot G_{3} \\
= & \left(T_{n-1}+T_{n-2}\right) \cdot G_{1}+\left(T_{n+1}\right) \cdot G_{2}+\left(T_{n}\right) \cdot G_{3}
\end{aligned}
$$

This completes the proof.

Now consider the sequence behavior for negative indices. To do so, we can describe properties involving the following set:

$$
\left(\ldots, G_{-n-1}, G_{-n}, G_{-n+1}, G_{-n+2}, G_{-n+3}, \ldots, G_{-3}, G_{-2}, G_{-1}, G_{0}, G_{1}, G_{1}, G_{2}, \ldots\right) .
$$

Therefore, from Definition 3, we will take:

$$
\begin{gathered}
n=0 \therefore G_{0}=G_{-2}+G_{-3}+G_{-4} \therefore G_{-4}=G_{0}-G_{-2}-G_{-3}=-G_{-2}-G_{-3} \\
G_{-4}=(-1) \cdot G_{-2}+(-1) \cdot G_{-3}=T_{-2} \cdot G_{-1}+\left(T_{-4}+T_{-5}\right) \cdot G_{-2}+T_{-4} \cdot G_{-3}
\end{gathered}
$$

To another value:

$$
n=-1 \therefore G_{-1}=G_{-3}+G_{-4}+G_{-5} \therefore G_{-5}=G_{-1}-G_{-3}-G_{-4}=G_{-1}-G_{-3}-\left(-G_{-2}-G_{-3}\right)=G_{-1}+G_{-2}
$$

Or, we can write

$$
G_{-5}=1 \cdot G_{-1}+1 \cdot G_{-2}+0 \cdot G_{-3}=T_{-3} \cdot G_{-1}+\left(T_{-5}+T_{-6}\right) \cdot G_{-2}+T_{-5} \cdot G_{-3} .
$$

For one more value, we have:

$$
\begin{aligned}
n & =-2 \therefore G_{-2}=G_{-4}+G_{-5}+G_{-6} \therefore G_{-6}=G_{-2}-G_{-4}-G_{-5}=G_{-2}-\left(-G_{-2}-G_{-3}\right)-\left(G_{-1}+G_{-2}\right) \\
& =-G_{-1}+G_{-2}+G_{-3} .
\end{aligned}
$$

Finally, we find:

$$
\begin{aligned}
G_{-6} & =(-1) \cdot G_{-1}+1 \cdot G_{-2}+1 \cdot G_{-3} \\
& =T_{-4} \cdot G_{-1}+\left(T_{-6}+T_{-7}\right) \cdot G_{-2}+T_{-6} \cdot G_{-3} .
\end{aligned}
$$

From the previous recursive procedure, we must find the following set 


$$
\left\{\begin{array}{l}
G_{-4}=T_{-2} \cdot G_{-1}+\left(T_{-4}+T_{-5}\right) \cdot G_{-2}+T_{-4} \cdot G_{-3} \\
G_{-5}=T_{-3} \cdot G_{-1}+\left(T_{-5}+T_{-6}\right) \cdot G_{-2}+T_{-5} \cdot G_{-3} \\
G_{-6}=T_{-4} \cdot G_{-1}+\left(T_{-6}+T_{-7}\right) \cdot G_{-2}+T_{-6} \cdot G_{-3} \\
G_{-7}=T_{-5} \cdot G_{-1}+\left(T_{-7}+T_{-8}\right) \cdot G_{-2}+T_{-7} \cdot G_{-3} \\
G_{-8}=T_{-6} \cdot G_{-1}+\left(T_{-8}+T_{-9}\right) \cdot G_{-2}+T_{-8} \cdot G_{-3} \\
G_{-9}=T_{-7} \cdot G_{-1}+\left(T_{-9}+T_{-10}\right) \cdot G_{-2}+T_{-9} \cdot G_{-3} \\
\vdots \\
G_{-n}=T_{-n+2} \cdot G_{-1}+\left(T_{-n}+T_{-n-1}\right) \cdot G_{-2}+T_{-n} \cdot G_{-3}
\end{array} .\right.
$$

Now, from the previous relationships, we will formulate the following theorem.

Theorem 4. For any integer $n \geq 0$ we will have the following general term:

$$
G_{-n}=T_{-n+2} \cdot G_{-1}+\left(T_{-n}+T_{-n-1}\right) \cdot G_{-2}+T_{-n} \cdot G_{-3} \cdot
$$

Proof. Some initial steps were indicated in the previous paragraphs, however, only to confirm our conjecture, we will perform the method of induction.

$$
G_{-n}=T_{-n+2} \cdot G_{-1}+\left(T_{-n}+T_{-n-1}\right) \cdot G_{-2}+T_{-n} \cdot G_{-3}
$$

and let us notice the following relation

$$
G_{n+2}=G_{n}+G_{n-1}+G_{n-2}
$$

and replacing the index $n$ with $-n$, it gives

$$
\begin{aligned}
G_{-n+2} & =G_{-n}+G_{-n-1}+G_{-n-2} \therefore G_{-n-2} \\
& =G_{-n+2}-G_{-n}-G_{-n-1} .
\end{aligned}
$$

In this way, we will add $G_{-n}$ to the expression described above, resulting in:

$$
\begin{aligned}
\left(G_{-n+2}-G_{-n}\right)-G_{-n-1} & =\left(G_{-n+2}-G_{-n-1}\right)-G_{-n} \\
& =\left(G_{-n+2}-G_{-n-1}\right)-T_{-n+2} \cdot G_{-1}-\left(T_{-n}+T_{-n-1}\right) \cdot G_{-2}-T_{-n} \cdot G_{-3} .
\end{aligned}
$$

On the left-hand side, we determine: $G_{-n-2}=G_{-n+2}-G_{-n}-G_{-n-1}$ while, on the right-hand side, we will develop the expression, noting that $G_{-n-1}=T_{-n+1} \cdot G_{-1}+\left(T_{-n-1}+T_{-n-2}\right) \cdot G_{-2}+T_{-n-1} \cdot G_{-3}$ and $G_{-n+2}=T_{-n+4} \cdot G_{-1}+\left(T_{-n+2}+T_{-n+1}\right) \cdot G_{-2}+T_{-n+2} \cdot G_{-3}$.

Likewise,

$$
\left\{\begin{array}{l}
G_{-n+2}=T_{-n+4} \cdot G_{-1}+\left(T_{-n+2}+T_{-n+1}\right) \cdot G_{-2}+T_{-n+2} \cdot G_{-3} \\
G_{-n-1}=T_{-n+1} \cdot G_{-1}+\left(T_{-n-1}+T_{-n-2}\right) \cdot G_{-2}+T_{-n-1} \cdot G_{-3} \\
G_{-n}=T_{-n+2} \cdot G_{-1}+\left(T_{-n}+T_{-n-1}\right) \cdot G_{-2}+T_{-n} \cdot G_{-3}
\end{array}\right.
$$

In the previous system, we evaluated:

$$
\begin{aligned}
G_{-n+2}-G_{-n-1}-G_{-n}= & \left(T_{-n+4}-T_{-n+1}-T_{-n+2}\right) \cdot G_{-1}+ \\
& +\left(T_{-n+2}-T_{-n}-T_{-n-1}+T_{-n+1}-T_{-n-1}-T_{-n-2}\right) \cdot G_{-2}+\left(T_{-n+2}-T_{-n-1}-T_{-n}\right) \cdot G_{-3}
\end{aligned}
$$

Finally, noting the following relationships

$$
\begin{aligned}
T_{-n} & =T_{-n+4}-T_{-n+1}-T_{-n+2}, \\
T_{-n-2} & =T_{-n+2}-T_{-n-1}-T_{-n}, \\
T_{-n-3} & =T_{-n+1}-T_{-n-1}-T_{-n-2},
\end{aligned}
$$


we can replace and find:

$$
\begin{aligned}
G_{-n-2} & =G_{-n+2}-G_{-n-1}-G_{-n} \\
& =T_{-n} \cdot G_{-1}+\left(T_{-n-2}+T_{-n-3}\right) \cdot G_{-2}+\left(T_{-n+2}-T_{-n+1}-T_{-n}\right) \cdot G_{-3}
\end{aligned}
$$

Thus, we determine that $G_{-n-2}=T_{-n} \cdot G_{-1}+\left(T_{-n-2}+T_{-n-3}\right) \cdot G_{-2}+T_{-n-2} \cdot G_{-3}$.

Corollary. For any integer, the following relations are valid:

(i) $\sum_{i=4}^{n} G_{i}=\left(\sum_{i=2}^{n-4} t_{i}+\sum_{i=1}^{n-3} t_{i}\right) \cdot G_{1}+\sum_{i=3}^{n-1} t_{i} \cdot G_{2}+\sum_{i=2}^{n-2} t_{i} \cdot G_{3}$,

(ii) $\sum_{i=4}^{n} G_{-i}=\sum_{i=2}^{n-1} t_{-i} \cdot G_{-1}+\left(\sum_{i=4}^{n} t_{-i}+\sum_{i=5}^{n+1} t_{-i}\right) \cdot G_{-2}+\sum_{i=4}^{n} t_{-i} \cdot G_{-3}$.

Proof. Consider the following identities and then add them up, so that the terms $G_{1}, G_{2}, G_{3}$ are highlighted.

$$
\left\{\begin{array} { l } 
{ G _ { 4 } = ( T _ { 2 } + T _ { 1 } ) \cdot G _ { 1 } + T _ { 3 } \cdot G _ { 2 } + T _ { 2 } \cdot G _ { 3 } } \\
{ G _ { 5 } = ( T _ { 1 } + T _ { 2 } ) \cdot G _ { 1 } + T _ { 4 } \cdot G _ { 2 } + T _ { 3 } \cdot G _ { 3 } } \\
{ G _ { 6 } = ( T _ { 2 } + T _ { 3 } ) \cdot G _ { 1 } + T _ { 5 } \cdot G _ { 2 } + T _ { 4 } \cdot G _ { 3 } } \\
{ G _ { 7 } = ( T _ { 3 } + T _ { 4 } ) \cdot G _ { 1 } + T _ { 6 } \cdot G _ { 2 } + T _ { 5 } \cdot G _ { 3 } } \\
{ \vdots } \\
{ G _ { n } = ( T _ { n - 4 } + T _ { n - 3 } ) \cdot G _ { 1 } + T _ { n - 1 } \cdot G _ { 2 } + T _ { n - 2 } \cdot G _ { 3 } }
\end{array} \quad \left\{\begin{array}{l}
G_{-4}=T_{-2} \cdot G_{-1}+\left(T_{-4}+T_{-5}\right) \cdot G_{-2}+T_{-4} \cdot G_{-3} \\
G_{-5}=T_{-3} \cdot G_{-1}+\left(T_{-5}+T_{-6}\right) \cdot G_{-2}+T_{-5} \cdot G_{-3} \\
G_{-6}=T_{-4} \cdot G_{-1}+\left(T_{-6}+T_{-7}\right) \cdot G_{-2}+T_{-6} \cdot G_{-3} \\
G_{-7}=T_{-5} \cdot G_{-1}+\left(T_{-7}+T_{-8}\right) \cdot G_{-2}+T_{-7} \cdot G_{-3} \\
\vdots \\
G_{-n}=T_{-n+2} \cdot G_{-1}+\left(T_{-n}+T_{-n-1}\right) \cdot G_{-2}+T_{-n} \cdot G_{-3}
\end{array}\right.\right.
$$

We will immediately have the identities foreseen in Theorems 3 and 4.

According to the Padovan $Q$-matrix [11], we have the Tridovan $Q$-matrix, with $T_{0}=0$, $T_{1}=1, T_{2}=0, T_{3}=1$,

$$
Q=\left[\begin{array}{llll}
0 & 1 & 0 & 0 \\
1 & 0 & 1 & 0 \\
1 & 0 & 0 & 1 \\
1 & 0 & 0 & 0
\end{array}\right]
$$

Theorem 5. Any term of the Tridovan sequence can be obtained by calculating the $n$-th power of $Q$, with $T_{0}=0, T_{1}=1, T_{2}=0, T_{3}=1$. This is represented by $Q^{n}$.

$$
Q^{n}=\left[\begin{array}{llll}
0 & 1 & 0 & 0 \\
1 & 0 & 1 & 0 \\
1 & 0 & 0 & 1 \\
1 & 0 & 0 & 0
\end{array}\right]^{n}=\left[\begin{array}{llll}
Q(1,1) & Q(1,2) & Q(1,3) & Q(1,4) \\
Q(2,1) & Q(2,2) & Q(2,3) & Q(2,4) \\
Q(3,1) & Q(3,2) & Q(3,3) & Q(3,4) \\
Q(4,1) & Q(4,2) & Q(4,3) & Q(4,4)
\end{array}\right], n \geq 3
$$

In order to simplify the terms visualization of the previous matrix, the calculation of each of these is presented in the following lines: 


$$
\begin{array}{ll}
Q^{n+1}(1,1)=T_{(n)}, & Q^{n+1}(3,1)=T_{(n-1)}+T_{(n-2)}, \\
Q^{n+1}(1,2)=T_{(n-1)}, & Q^{n+1}(3,2)=T_{(n-2)}+T_{(n-3)}, \\
Q^{n+1}(1,3)=T_{(n-2)}, & Q^{n+1}(3,3)=T_{(n-3)}+T_{(n-4)}, \\
Q^{n+1}(1,4)=T_{(n-3)}, & Q^{n+1}(3,4)=T_{(n-4)}+T_{(n-5)}, \\
Q^{n+1}(2,1)=T_{(n-1)}+T_{(n-2)}+T_{(n-3)}, & Q^{n+1}(4,1)=T_{(n-1)}, \\
Q^{n+1}(2,2)=T_{(n-2)}+T_{(n-3)}+T_{(n-4)}, & Q^{n+1}(4,2)=T_{(n-2)}, \\
Q^{n+1}(2,3)=T_{(n-3)}+T_{(n-4)}+T_{(n-5)}, & Q^{n+1}(4,3)=T_{(n-3)}, \\
Q^{n+1}(2,4)=T_{(n-4)}+T_{(n-5)}+T_{(n-6)}, & Q^{n+1}(4,4)=T_{(n-4)} .
\end{array}
$$

Proof. Using the principle of finite induction to prove the theorem. We have that for $n=n+1$ :

$$
Q^{n+1}=Q^{n} \cdot Q
$$

$$
Q^{n}=\left[\begin{array}{llll}
Q(1,1) & Q(1,2) & Q(1,3) & Q(1,4) \\
Q(2,1) & Q(2,2) & Q(2,3) & Q(2,4) \\
Q(3,1) & Q(3,2) & Q(3,3) & Q(3,4) \\
Q(4,1) & Q(4,2) & Q(4,3) & Q(4,4)
\end{array}\right]^{n} \cdot\left[\begin{array}{llll}
0 & 1 & 0 & 0 \\
1 & 0 & 1 & 0 \\
1 & 0 & 0 & 1 \\
1 & 0 & 0 & 0
\end{array}\right]
$$

It is assumed that each element of the Tridovan $Q$-matrix is defined as:

$$
\begin{aligned}
& Q^{n+1}(1,1)=T_{(n-1)}+T_{(n-2)}+T_{(n-3)}=T_{(n+1)}, \\
& Q^{n+1}(1,2)=T_{(n)}=T_{(n+1)-1}, \\
& Q^{n+1}(1,3)=T_{(n-1)}=T_{(n+1)-2}, \\
& Q^{n+1}(1,4)=T_{(n-2)}=T_{(n+1)-3}, \\
& Q^{n+1}(2,1)=T_{(n-2)}+T_{(n-3)}+T_{(n-4)}+T_{(n-3)}+T_{(n-4)}+T_{(n-5)}+T_{(n-4)}+T_{(n-5)}+T_{(n-6)} \\
& =T_{(n)}+T_{(n-1)}+T_{(n-2)}=T_{(n+1)-1}+T_{(n+1)-2}+T_{(n+1)-3}, \\
& Q^{n+1}(2,2)=T_{3(n-1)}+T_{3(n-2)}+T_{(n-3)}=T_{(n+1)-2}+T_{(n+1)-3}+T_{(n+1)-4}, \\
& Q^{n+1}(2,3)=T_{3(n-2)}+T_{(n-3)}+T_{(n-4)}=T_{(n+1)-3}+T_{(n+1)-4}+T_{(n+1)-5}, \\
& Q^{n+1}(2,4)=T_{(n-3)}+T_{(n-4)}+T_{3(n-5)}=T_{3(n+1)-4}+T_{3(n+1)-5}+T_{3(n+1)-6}, \\
& Q^{n+1}(3,1)=T_{(n-2)}+T_{(n-3)}+T_{(n-3)}+T_{(n-4)}+T_{(n-4)}+T_{(n-5)}=T_{(n)}+T_{(n-1)}=T_{(n+1)-1}+T_{(n+1)-2} \text {, } \\
& Q^{n+1}(3,2)=T_{(n-1)}+T_{(n-2)}=T_{(n+1)-2}+T_{(n+1)-3}, \\
& Q^{n+1}(3,3)=T_{(n-2)}+T_{(n-3)}=T_{(n+1)-3}+T_{(n+1)-4}, \\
& Q^{n+1}(3,4)=T_{(n-3)}+T_{(n-4)}=T_{(n+1)-4}+T_{(n+1)-5}, \\
& Q^{n+1}(4,1)=T_{(n-2)}+T_{(n-3)}+T_{3(n-4)}=T_{3(n)}=T_{3(n+1)-1}, \\
& Q^{n+1}(4,2)=T_{(n-1)}=T_{(n+1)-2}, \\
& Q^{n+1}(4,3)=T_{(n-2)}=T_{(n+1)-3}, \\
& Q^{n+1}(4,4)=T_{(n-3)}=T_{(n+1)-4} \text {. }
\end{aligned}
$$

According to Definition 5, we can observe the following matrices: 


$$
\begin{aligned}
Q^{6} & =\left[\begin{array}{llll}
4 & 2 & 2 & 1 \\
5 & 4 & 2 & 2 \\
4 & 3 & 2 & 1 \\
2 & 2 & 1 & 1
\end{array}\right]=\left[\begin{array}{cccc}
T_{6} & T_{5} & T_{4} & T_{3} \\
T_{5}+T_{4}+T_{3} & T_{4}+T_{3}+T_{2} & T_{3}+T_{2}+T_{1} & T_{2}+T_{1}+T_{0} \\
T_{5}+T_{4} & T_{4}+T_{3} & T_{3}+T_{2} & T_{2}+T_{1} \\
T_{5} & T_{4} & T_{3} & T_{2}
\end{array}\right], \\
Q^{7} & =\left[\begin{array}{llll}
5 & 4 & 2 & 2 \\
8 & 5 & 4 & 2 \\
6 & 4 & 3 & 2 \\
4 & 2 & 2 & 1
\end{array}\right]=\left[\begin{array}{cccc}
T_{7} & T_{6} & T_{5} & T_{4} \\
T_{6}+T_{5}+T_{4} & T_{5}+T_{4}+T_{3} & T_{4}+T_{3}+T_{2} & T_{3}+T_{2}+T_{1} \\
T_{6}+T_{5} & T_{5}+P_{4} & T_{4}+T_{3} & T_{3}+T_{2} \\
T_{6} & T_{5} & T_{4} & T_{3}
\end{array}\right] .
\end{aligned}
$$

Similarly, we observe:

$$
\begin{aligned}
Q^{-1} & =\left[\begin{array}{rrrr}
0 & 0 & 0 & 1 \\
1 & 0 & 0 & 0 \\
0 & 1 & 0 & -1 \\
0 & 0 & 1 & -1
\end{array}\right]=\left[\begin{array}{cccc}
T_{-1} & T_{-2} & T_{-3} & T_{-4} \\
T_{-2}+T_{-3}+T_{-4} & T_{-3}+T_{-4}+T_{-5} & T_{-4}+T_{-5}+T_{-6} & T_{-5}+T_{-6}+T_{-7} \\
T_{-2}+T_{-3} & T_{-3}+T_{-4} & T_{-4}+T_{-5} & T_{-5}+T_{-6} \\
T_{-2} & T_{-3} & T_{-4} & T_{-5}
\end{array}\right], \\
Q^{-2} & =\left[\begin{array}{rrrr}
0 & 0 & 1 & -1 \\
0 & 0 & 0 & 1 \\
1 & 0 & -1 & 1 \\
0 & 1 & -1 & 0
\end{array}\right]=\left[\begin{array}{cccc}
T_{-2} & T_{-3} & T_{-4} & T_{-5} \\
T_{-3}+T_{-4}+T_{-5} & T_{-4}+T_{-5}+T_{-6} & T_{-5}+T_{-6}+T_{-7} & T_{-6}+T_{-7}+T_{-8} \\
T_{-3}+T_{-4} & T_{-4}+T_{-5} & T_{-5}+T_{-6} & T_{-6}+T_{-7} \\
T_{-3} & T_{-4} & T_{-5} & T_{-6}
\end{array}\right] .
\end{aligned}
$$

Theorem 6. The determinant calculation of the Tridovan Q-matrix powered to the $n$-th power is always equal to the number 1, namely, $\operatorname{det} Q^{n}=1$.

Proof.

$$
\operatorname{det} Q=1, \quad \operatorname{det}\left(Q^{n}\right)=\operatorname{det}(Q)^{n}=1 .
$$

Theorem 7. For any natural numbers $m$ and $n, 3 \leq m<n$, we have the following relation:

$$
T_{n}=T_{m} \cdot T_{n-m}+T_{m-1} \cdot\left(T_{n-m-1}+T_{n-m-2}+T_{n-m-3}\right)+T_{m-2} \cdot\left(T_{n-m-1}+T_{n-m-2}\right)+T_{m-3} \cdot T_{n-m-1} .
$$

Proof.

$Q^{n}=Q^{m} \cdot Q^{n-m}$

$$
\begin{aligned}
& Q^{n}=\left[\begin{array}{cccc}
T_{n} & T_{n-1} & T_{n-2} & T_{n-3} \\
T_{n-1}+T_{n-2}+T_{n-3} & T_{n-2}+T_{n-3}+T_{n-4} & T_{n-3}+T_{n-4}+T_{n-5} & T_{n-4}+T_{n-5}+T_{n-6} \\
T_{n-1}+T_{n-2} & T_{n-2}+T_{n-3} & T_{n-3}+T_{n-4} & T_{n-4}+T_{n-5} \\
T_{n-1} & T_{n-2} & T_{n-3} & T_{n-4}
\end{array}\right] \\
& =\left[\begin{array}{cccc}
T_{m} & T_{m-1} & T_{m-2} & T_{m-3} \\
T_{m-1}+T_{m-2}+T_{m-3} & T_{m-2}+T_{m-3}+T_{m-4} & T_{m-3}+T_{m-4}+T_{m-5} & T_{m-4}+T_{m-5}+T_{m-6} \\
T_{m-1}+T_{m-2} & T_{m-2}+T_{m-3} & T_{m-3}+T_{m-4} & T_{m-4}+T_{m-5} \\
T_{m-1} & T_{m-2} & T_{m-3} & T_{m-4}
\end{array}\right] \\
& \cdot\left[\begin{array}{cccc}
T_{n-m} & T_{n-m-1} & T_{n-m-2} & T_{n-m-3} \\
T_{n-m-1}+T_{n-m-2}+T_{n-m-3} & T_{n-m-2}+T_{n-m-3}+T_{n-m-4} & T_{n-m-3}+T_{n-m-4}+T_{m-5} & T_{n-m-4}+T_{n-m-5}+T_{n-m-6} \\
T_{n-m-1}+T_{n-m-2} & T_{n-m-2}+T_{n-m-3} & T_{n-m-3}+T_{n-m-4} & T_{n-m-4}+T_{n-m-5} \\
T_{n-m-1} & T_{n-m-2} & T_{n-m-3} & T_{n-m-4}
\end{array}\right]
\end{aligned}
$$


$T_{n}=T_{m} \cdot T_{n-m}+T_{m-1} \cdot\left(T_{n-m-1}+T_{n-m-2}+T_{n-m-3}\right)+T_{m-2} \cdot\left(T_{n-m-1}+T_{n-m-2}\right)+T_{m-3} \cdot T_{n-m-1} \cdot$

If $m=3$,

$$
\begin{aligned}
& T_{n}=T_{3} \cdot T_{n-3}+T_{2} \cdot(\underbrace{T_{n-4}+T_{n-5}+T_{n-6}}_{P_{n-2}})+T_{1} \cdot\left(T_{n-4}+T_{n-5}\right)+T_{0} \cdot T_{n-4} \\
& T_{n}=T_{3} \cdot T_{n-3}+T_{2} \cdot T_{n-2}+T_{1} \cdot\left(T_{n-4}+T_{n-5}\right)+T_{0} \cdot T_{n-4} \quad \because T_{0}=1 ; T_{1}=0 ; T_{2}=T_{3}=1 \\
& T_{n}=T_{n-2}+T_{n-3}+T_{n-4} .
\end{aligned}
$$

\section{Conclusion}

Based on the studies carried out on this Tribonacci Sequence, initially studied by Feinberg [5], and then continued by Koshy [7], we can investigate and establish new identities for the Tridovan numbers.

In a similar way as for the Fibonacci extensions [2, 3], investigative and historical studies of this sequence are made so that it is possible to obtain the Tridovan Sequence, and thus discover new properties related to it. Based on the previous considerations, we emphasize that this work presents sufficient elements to characterize the general objective scope of the research. The new identities of the Tridovan Sequence, its generalization and the behavior of the terms for the positive and negative indices were presented. Besides that, it was still possible to obtain the Tridovan $Q$-matrix, so that it is possible to find the terms of the sequence without the use of the recurrence formula [12].

We can conclude that these Padovan numbers are in initial study and discoveries of relations and properties and more research and studies on the subject are required.

\section{References}

[1] Alsina, C., \& Nelsen, R. (2015). A Mathematical Space Odyssey: Solid Geometry in the 21st Century (Dolciani Mathematical Expositions). American Mathematical Society, Edition: UK ed.

[2] Alves, F. R. V. (2015). Sequência Generalizada de Fibonacci e Relações com o número Áureo. Boletim Cearense de Educação e História da Matemática. Ceará, 2 (6): 30-36.

[3] Alves, F. R. V. (2015). Sobre a evolução histórica do modelo de Fibonacci: A classe das funções hiperbólicas de Fibonacci - FHF. Vidya, 35 (1): 133-146.

[4] Cereceda, L. J. (2015). Binet's formula for generalized Tribonacci numbers. International Journal of Mathematical Education in Science and Technology, 46 (8), 1235-1243.

[5] Feinberg, M. (1963). Fibonacci-Tribonacci. The Fibonacci Quarterly, [S.1.], 1 (3), 209-222.

[6] Iliopoulos, V. (2015). The plastic number and its generalized polynomial. Cogent Mathematics, 2, 1-6. 
[7] Koshy, T. (2011). Fibonacci and Lucas Numbers with Applications. New York: John Wiley and Sons.

[8] Marohnic, L., \& Strmecki, T. (2013). Plastic number: Construction and applications. Advanced Research in Scientific Areas, 2, 1523-1528.

[9] Miller, M. D. (1971). On Generalized Fibonacci Numbers. The American Mathematical Monthly, 78 (10), 1108-1109.

[10] Shannon, A. G., Horadam, A. F., \& Anderson, P. G. (2006). The auxiliary equation associated with the plastic number. Notes on Number Theory and Discrete Mathematics, $12(1), 1-12$.

[11] Sokhuma, K. (2013). Padovan $Q$-matrix and the generalized relations. Applied Mathematical Sciences. 7 (56), 2777-2780.

[12] Stewart, I. (2000). L'Univers des Nombres. Paris: Belin pour la Science. 